Proc. Indian Acad. Sci. (Earth Planet. Sci.), Vol. 97, No. 2, December 1988, pp. 173-181.

(C) Printed in India.

\title{
Spectral width parameter for wind-generated ocean waves
}

\author{
C V K PRASADA RAO \\ Naval Physical and Oceanographic Laboratory, Cochin 682004, India \\ MS received 5 December 1986; revised 8 June 1988
}

\begin{abstract}
An evaluation of the spectral width parameter for wind-generated ocean waves is presented based on the analysis of wave data obtained through the ship-borne wave recorder fitted to RV Gaveshani. The coverage of wave data includes deep as well as shallow waters on the east and west coasts of India. Considering that the spectral width parameter $E_{0}$ derived from Tucker's wave analysis provides a reasonably accurate estimate, a comparative study has been made for the other band-width definitions available in the literature. The results indicate some bias in the estimation of spectral width information using higher-order moments, e.g. $\varepsilon_{s}$ and $v$, and with the alteration of spectral forms that are usually associated with multiple- or single-peaked wave spectra. Such a bias is not seen in the case of Goda's peakedness parameter $Q_{p}$ with variation of spectral form. The effect of the ratio of the high-frequency cut-off to the spectral peak period on $\varepsilon_{s} v$ and $Q_{p}$ is also investigated.
\end{abstract}

Keywords. Spectral width parameter; ship-borne wave recorder.

\section{Introduction}

Longuet-Higgins $(1952,1975)$ derived theoretical distributions of wave heights as well as the joint distribution of wave heights and periods based on the assumption that the wave frequencies lie in a narrow band with random phase values. In general it is accepted that the sea surface elevations follow a 'Gaussian' distribution and the wave heights follow a 'Rayleigh' distribution. These theoretical concepts have been tested earlier using a few wave observations made off the Indian coast (Dattatri 1973; Dattatri et al 1976, 1979; John 1977; Narasimhan and Deo 1981; Fernandes et al 1981; Baba and Harish 1985). Since the narrow-band criterion is adopted for theoretical considerations, it is essential to quantify the spectra band-width for ocean waves for testing and validation of the theory against observations. Thus the spectral width parameter assumes importance for ocean wave studies as it provides a clue to the composition of sea state, viz. 'sea' and 'swell' conditions. The shape of the wave engery spectrum is also determined by using spectral width information and often the estimates of wave spectral shapes are considered important for ocean engineering applications. Attempts are therefore made in this investigation to study the spectral width parameter in detail by analysing some wave data collected off the east and west coasts of India.

\section{Analytical consideration}

From the zero-upcrossing method of wave analysis (Tucker 1963; Draper 1967) one can 
derive the spectral width parameter $\varepsilon_{0}$ using the formula

$$
\varepsilon_{0}=\left[1-\left(T_{c} / T_{z}\right)^{2}\right]^{1 / 2},
$$

where $T_{z}$ and $T_{c}$ are the mean zero-upcrossing period and the mean crest period respectively. The parameter $\varepsilon_{0}$ varies from 0 to 1 depending on the prevailing sea state, and $\varepsilon_{0} \rightarrow 1$ (approaching unity) implies broad-band wave conditions with smaller waves (short-period) riding on top of the larger waves (long-period). On the other hand $\varepsilon_{0} \rightarrow 0$ represents narrow-band characteristics which can be attributed to the presence of more regular waves, i.e. 'swell'. The spectral width values obtained through (1) can be considered as fairly accurate as careful estimations were made of $T_{z}$ and $T_{c}$ from the wave records.

However, it is often viewed by many users of wave information that the wave parameters derived by the Tucker/Draper method are just not adequate for complete description of the sea state and the use of spectral techniques is recommended for several coastal and offshore engineering works. In the wave spectrum approach the required significant wave parameters are derived from spectral moments and spectral density estimates. Any $n$th moment can be computed using the relationship

$$
m_{n}=\int_{\mathrm{LF}}^{\mathrm{HF}} f^{n} s(f) \mathrm{d} f,
$$

where LF and HF are lower and higher cut-off frequencies, and $f$ and $s(f)$ are the frequency and the corresponding spectral density estimates. The zeroth $\left(m_{0}\right), 1$ st $\left(m_{1}\right)$, 2nd $\left(m_{2}\right)$, and 4th $\left(m_{4}\right)$ are the most commonly used moments for computation of wave parameters. The moment $m_{0}$ is referred to as the variance of sea surface and is proportional to the total energy of a wave spectrum.

Cartwright and Longuet-Higgins (1956) and Longuet-Higgins (1975) have suggested the following relationships for obtaining the values of spectral width parameter from the moments:

$$
\begin{aligned}
\varepsilon_{s} & =\left[\left(m_{0} m_{4}-m_{2}^{2}\right) / m_{0} m_{4}\right]^{1 / 2}, \\
v & =\left[\left(m_{2} m_{0}-m_{1}^{2}\right) / m_{1}^{2}\right]^{1 / 2} .
\end{aligned}
$$

Both $\varepsilon_{s}$ and $v$ have the same range of numerical values as $\varepsilon_{0}$. The parameter $\varepsilon_{s}$ is also known as the spectral width parameter but $v$ is sometimes referred to as 'spectral narrowness parameter' in the literature (Rye 1976). Goda (1970) proposed another parameter called the 'peakedness parameter', defined as

$$
Q_{p}=\left(2 / m_{0}^{2}\right) \int_{L F}^{\mathrm{HF}} f[s(f)]^{2} \mathrm{~d} f .
$$

Unlike the other three parameters, i.e. $\varepsilon_{0}, \varepsilon_{s}$ and $v$, the numerical values of $Q_{p}$ are normally more than 1.0 and a larger magnitude implies a more sharply peaked wave spectrum (or narrow-band condition) and vice versa.

\section{Materials and methods}

The data used for this study were collected on board RV Gaveshani during two of her cruises-the 79 th and the $82 \mathrm{nd}$ - using a ship-borne wave recording system (IOS 
Model 5254, Institute of Oceanographic Sciences, UK) installed on the vessel. The 79 th cruise was conducted in the Bay of Bengal during the period 24th September to 4th October 1980. The wave measurements were made in this cruise at two locations south of Visakhapatnam, on the east coast of India, where the station depths are 250 and $500 \mathrm{~m}$. Vethamony et al $(1982,1984)$ had processed the wave data collected in this cruise following spectral and statistical methods (zero-upcrossing analysis). Data on spectral width information as well as various other wave statistics, e.g. wave heights, periods, etc., were presented (tables 1 and 2 of Vethamony et al 1982). For this investigation the author selected 59 wave records from the above for extracting necessary data on spectral width parameters. Apart from these data another 20 wave records collected during the 82 nd cruise between 12 th and 15 th November 1980 were also utilized for this study. The locations of wave measurements in this case lie in the shallow as well as deep waters off Goa, on the west coast of India. These data were analysed by the author (Prasada Rao 1984, 1985). The relevant data on spectral width parameters from the author's earlier works are also incorporated in this study. Thus, in total, 79 wave records have been considered for this investigation.

Regarding the methods of wave data analysis adopted, the following points may be noted. Each wave record is of approximately $15 \mathrm{~min}$ duration. For spectral analysis, the data were digitized with a sampling interval $\Delta t$ of $1 \mathrm{~s}$. An auto-correlation method was employed and a 'Hanning' window was used for smoothing raw spectral estimates (Bendat and Piersol 1971). As $\Delta t=1 \mathrm{~s}$ the Nyquist frequency $(1 / 2 \Delta t)$ is $0.5 \mathrm{~Hz}$. But due to the limitations of the ship-borne wave recorder used in the study, the lower limiting period was fixed as $3 \mathrm{~s}$ or $0.33 \mathrm{~Hz}$. It may be noted that the wave sensors were fitted to the ship's hull at $2 \mathrm{~m}$ below the water surface and very short period waves, i.e. waves of period $<3 \mathrm{~s}$, are therefore usually disregarded. Similarly the upper limiting wave period was chosen as $25 \mathrm{~s}$ from the point of instrument sensitivity. Spectral moments were computed (2) and these inputs were used for deriving values of $\varepsilon_{s}, v$ and $Q_{p}$. The band-width parameter $\varepsilon_{0}$ was arrived at following the zero-upcrossing method of wave analysis (Tucker 1963; Draper 1967).

\section{Results and discussion}

From section 2 one can see that, except $\varepsilon_{0}$ the spectral width parameters are based on moments. Spectral moments in general and higher-order moments in particular are somewhat unstable owing to their dependence on several factors, viz. energy density values on the high-frequency side of the spectrum, cut-off frequency choice, etc. These are again related to the accuracy of the instrument and the data sampling interval used for any study. On the other hand, as the $\varepsilon_{0}$ is defined based on the number of zeroupcrossings $N_{z}$ and the number of crests $N_{c}$ for a given wave record, this parameter is relatively better. Goda (1974) discussed these aspects in detail. Similar arguments which give more weightage to $\varepsilon_{0}$ compared to other spectral width definitions might be seen from Haring et al (1976). Therefore an attempt is made to evaluate the spectral width parameter values of $\varepsilon_{s}, v$ and $Q_{p}$ by comparing the same with those of $\varepsilon_{0}$.

Spectral analysis of wave data revealed the following aspects. By and large the spectra consisted of either a single peak or multiple peaks. The prirrary spectral peaks are noticed on the low-frequency side of the spectrum approximately around $0.06 / 0.07 \mathrm{~Hz}$. These peaks are mostly associated with significant amounts of wave energies compared to the high-frequency parts of the wave spectra. One probable 
reason for the predominant occurrence of low-frequency peaks could be the presence of 'swells'. The subsequent peaks other than 'swell' peaks might have been caused by the locally generated 'seas'. Thus the actual shape of the spectrum depends on the sea state. Since different types of spectral forms are encountered the observed spectra are classified as follows. Spectra possessing conspicuously a single peak represent the narrow-band condition and those with two or multiple peaks represent the broad-band condition. Some typical wave spectra identified for narrow-band and broad-band conditions are shown in figure $1(a, b)$. The classification was based on visual examination of spectral shapes.

Comparison of $\varepsilon_{0}$ and $\varepsilon_{s}$ is given in figure $2 \mathrm{a}$. From this it can be seen that $\varepsilon_{s}$ values are higher for narrow-band spectra and lower for broad-band spectra compared to $\varepsilon_{0}$. Earlier investigators found that $\varepsilon_{s}$ was either higher or lower than $\varepsilon_{0}$. Hoffman and Karst (1975) found that $\varepsilon_{s}$ estimates were higher than $\varepsilon_{0}$ by $15-20 \%$. Results obtained by Dattatri et al (1979) show that $\varepsilon_{s}$ values were consistently lower compared to $\varepsilon_{0}$. On the contrary Chakrabarty and Snider (1974), Haring et al (1976) and

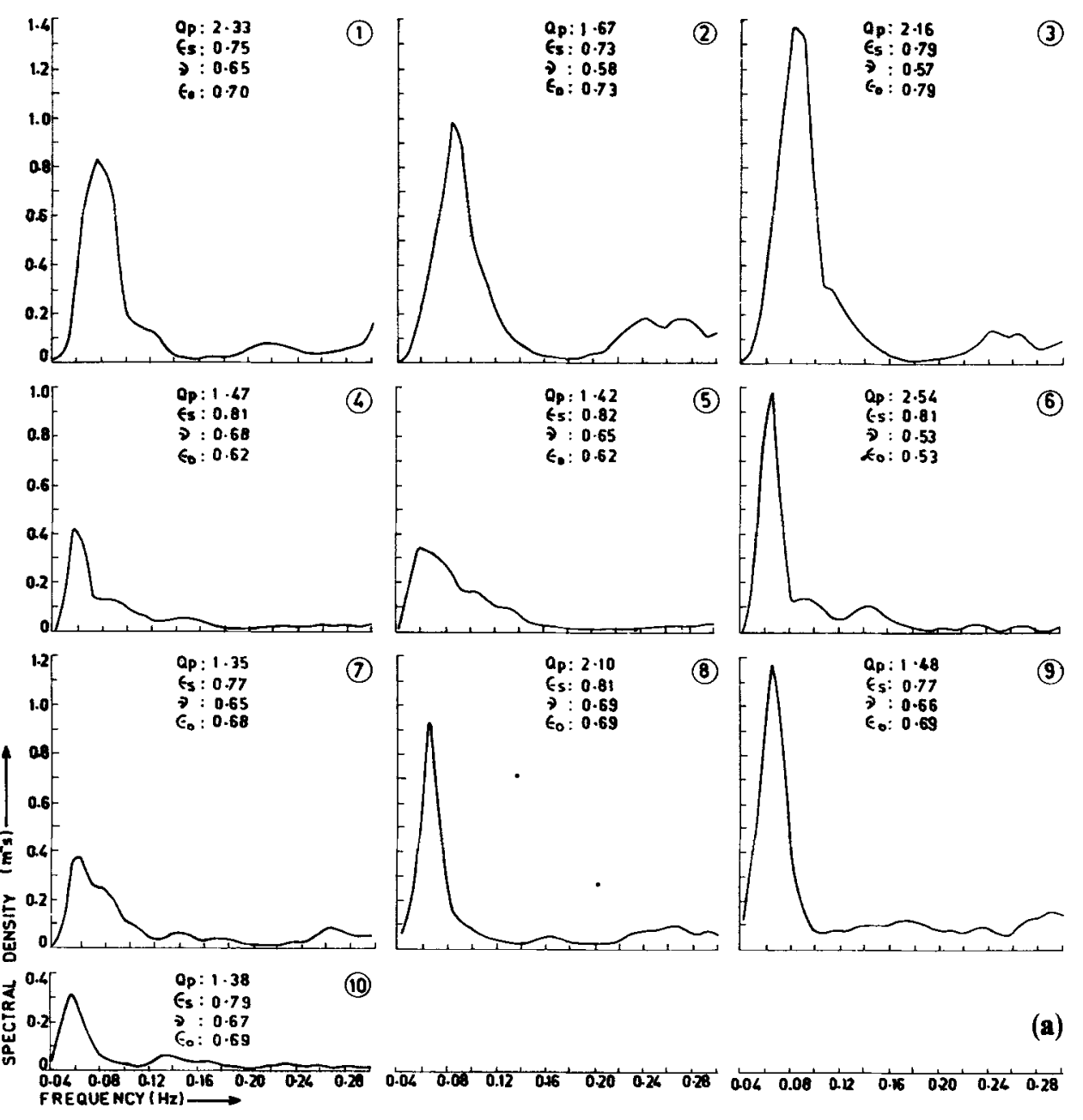



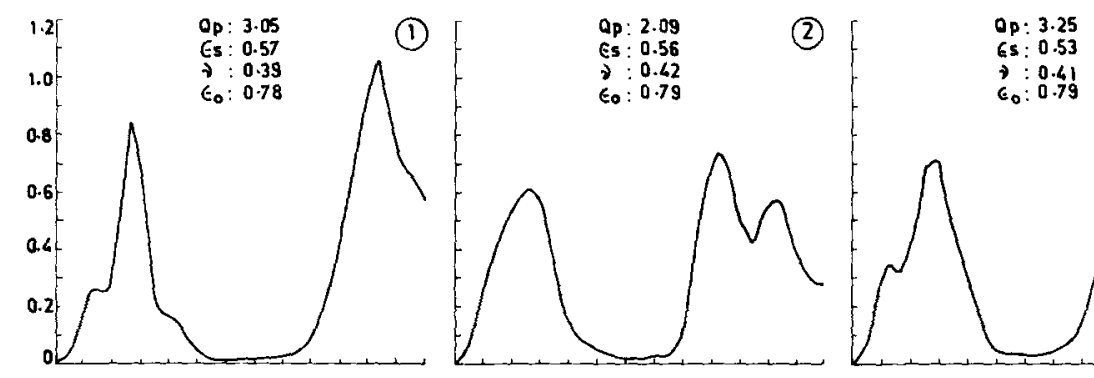

(3)

7
$\epsilon_{0}: 0.41$

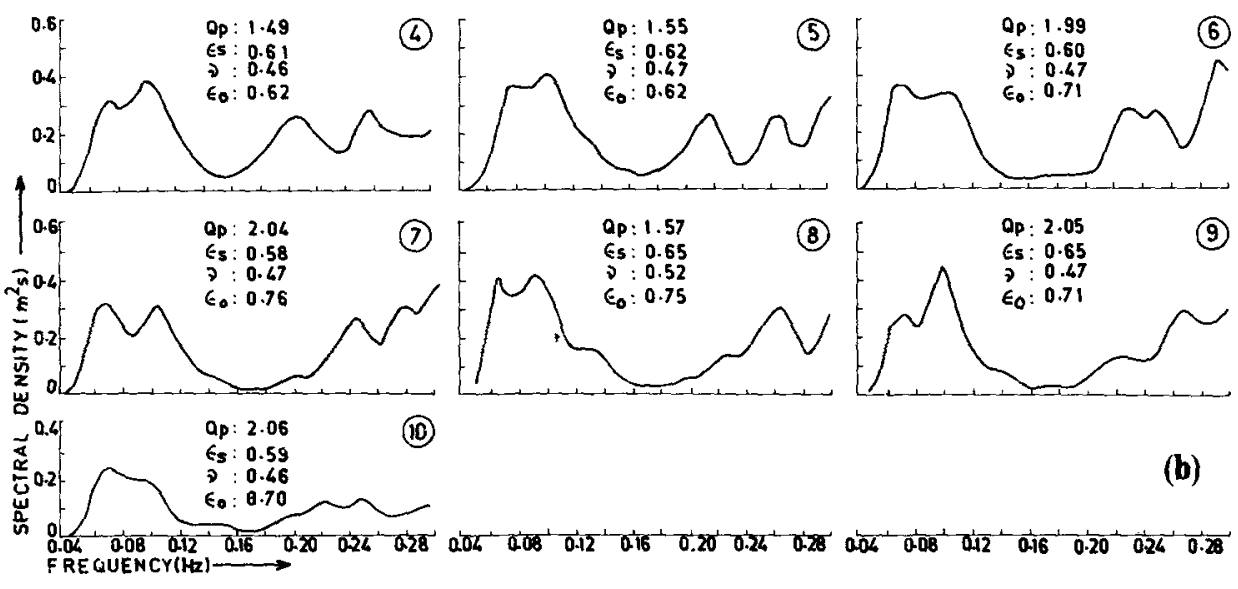

Figure 1. Some typical wave spectra consisting of (a) single peak and (b) multiple peaks.

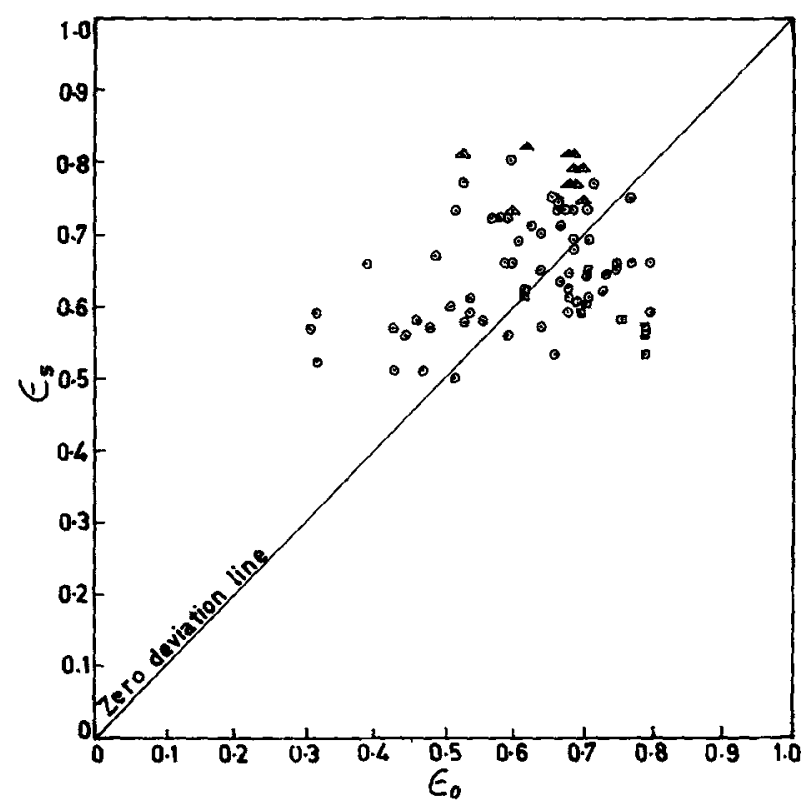

(a) 


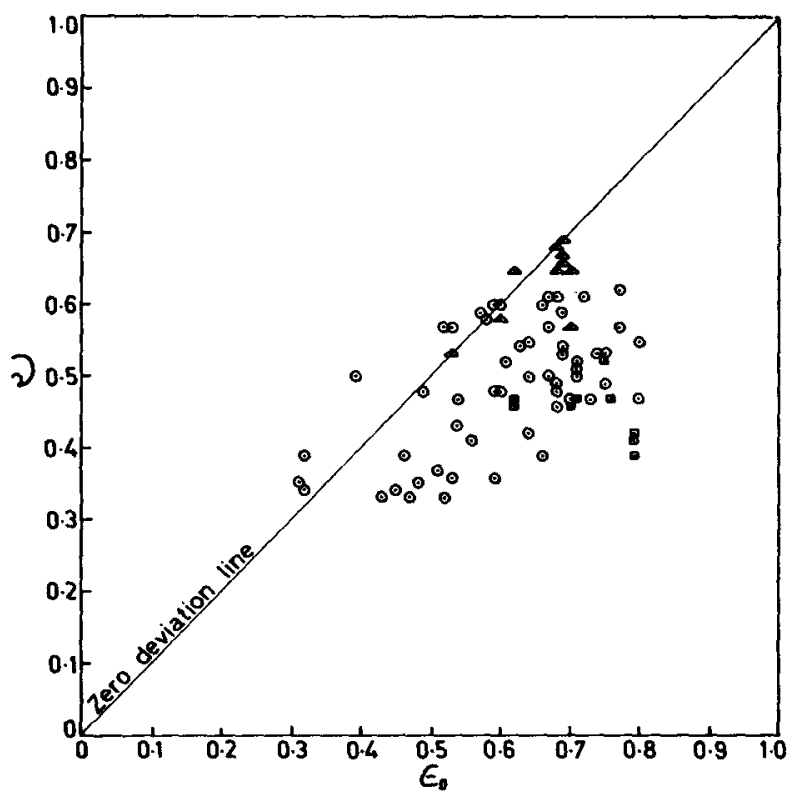

(b)

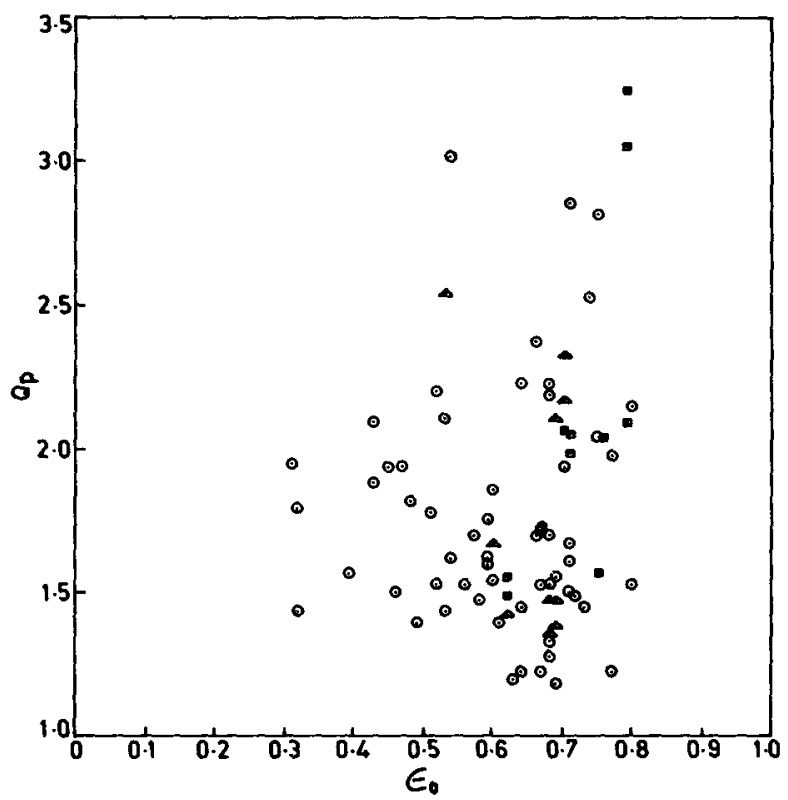

(c)

Figure 2. Comparison of the estimates of spectral width parameter obtained from the zero-upcrossing method with those derived from spectral analysis. Data points ' $\Delta$ ' represent single-peaked spectra (as in figure 1a), ' $\square$ ' represent multiple-peaked spectra (as in figure Ib) and ' $O$ ' represent the rest of the observations. (a) $\varepsilon_{0}$ vs $\varepsilon_{s}$ (b) $\varepsilon_{0}$ vs $v$ and (c) $\varepsilon_{0}$ vs $Q_{p}$. 
Fernandes et al (1981) pointed out that no consistent relation was observed between $\varepsilon_{0}$ and $\varepsilon_{s}$. Vethamony et al (1984) reported that $\varepsilon_{s}$ was greater for $45 \%$ of spectra (out of 61 observations) and the same was found to be lower for spectra with multiple peaks. Thus there exist divergent opinions as regards $\varepsilon_{s}$ estimates but the main debate is whether it is an overestimate or an underestimate. Nevertheless it is evident from the discussion presented above that $\varepsilon_{s}$ is less reliable and shows various anomalies. The present study substantiates this point and further following inferences may be drawn. Apparently $\varepsilon_{s}$ does not necessarily give overestimate. Overestimation through $\varepsilon_{s}$ might be expected for the narrow-band condition or for spectra with a single peak which might occur because of the presence of a 'swell'. Multiple peaks in wave spectra can also occur when the growth of waves takes place along with existing swells and $\varepsilon_{s}$ in such a case may cause underestimation of spectral width.

Figure $2 b$ presents a comparison between the spectral narrowness parameter $v$ and $\varepsilon_{0}$. Though the trend in the figure confirms that $v$ is in general less compared to $\varepsilon_{0}$, it is worth mentioning that the estimates of this parameter are relatively better for narrowband wave spectra. Hence $v$ offers a better estimate than $\varepsilon_{s}$ in the case of narrow-band conditions. However, the spectral narrowness parameter $v$ seems to fail to give correct estimates for broad-band spectra and its value is found to be reduced by almost a half of the value of $\varepsilon_{0}$ for some such cases. Dattatri (1979) also reported that $v$ was approximately $0.5 \varepsilon_{0}$. But it must be noted that such an approximation might be invalid under narrow-band conditions.

Among the spectrally derived band-width parameters, Goda's peakedness parameter $Q_{p}$ is more widely accepted. The reasons for this are: (i) $Q_{p}$ is more reliable as stable quantities like $m_{0}$ and $s(f)$ are used for its computation, and (ii) it is independent of cut-off frequency choice. The peakedness parameter is a measure of the sharpness of the wave spectrum and hence higher values of $Q_{p}$ imply more sharply peaked spectra, and vice versa. Further one can expect the higher values of $Q_{p}$ are associated with narrow-band wave spectra and the lower values of $Q_{p}$ with broad-band wave spectra. This aspect has been studied by comparing $Q_{p}$ and $\varepsilon_{0}$ (figure $2 \mathrm{c}$ ). The data reveal this type of trend $\left(\varepsilon_{0} \propto 1 / Q_{p}\right)$ only for $Q_{p} \leqslant 2$; above this threshold such a relation is not seen between these two parameters. However, this needs further investigation. On the positive side, the variation in $Q_{p}$ does not seem to depend on the narrow-band or wideband criterion. It may be considered as a plus point as far as $Q_{p}$ is concerned. Moreover, less dependence of $Q_{p}$ on spectral form is also evident from figure $1(\mathrm{a}, \mathrm{b})$.

The selection of cut-off frequency for spectral computations is also considered important as it influences estimates of the spectral. width parameter. The nondimensional frequency values are obtained by taking the ratio of high-frequency cut-off (CF) to the spectral peak frequency $\left(f_{p}\right)$. These are compared with $\varepsilon_{s}, v$ and $Q_{p}$ in figure 3. Both $\varepsilon_{s}$ and $v$ show clear indication of an increasing trend as the $C F / f_{p}$ ratio increases. Thus, for a given wave spectrum, by changing the value of CF one may obtain different results for $\varepsilon_{s}$ as well as $v$. Hence $Q_{p}$ is better as it shows less dependence on $\mathrm{CF} / f_{p}$. These findings are in agreement with those of Rye and Svee (1976) and Haring et al (1976). Rye and Svee (1976) have opined that $Q_{p}$ is a more convenient parameter for characterizing the spectral distribution. They also found that $Q_{p}$ gives a linear relationship with the JONSWAP overshoot parameter or the peak enhancement factor $\gamma$ and, unlike $\varepsilon_{s}$ or $v$, it can distinguish better between a very sharply peaked JONSWAP spectrum $(\gamma=7)$ and a Pierson-Moskowitz-type spectrum $(\gamma=1)$. 


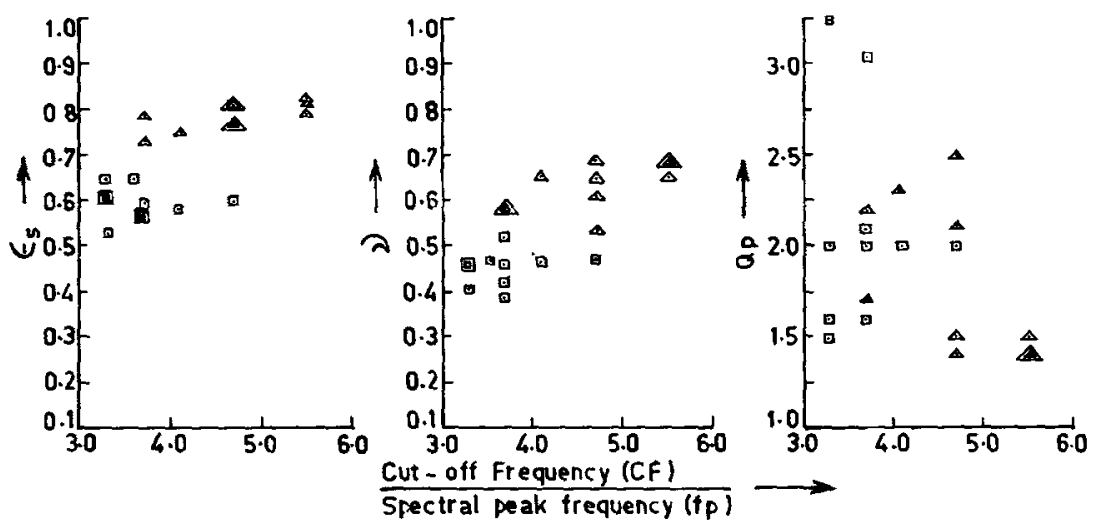

Figure 3. Influence of high-frequency cut-off (CF) on the spectral width estimates $v, \varepsilon_{s}$ and $Q_{p}$. CF is normalized to the spectral peak frequency, $f_{p}$. Data points ' $\Delta$ ' and ' $\square$ ' are as in figure 2.

\section{Summary and conclusions}

Spectral estimates of band-width parameters for ocean waves largely appear to be influenced by the nature of the wave spectrum. The occurrence of multiple peaks in spectra can alter the estimates and the effect of the same is very significant for $\varepsilon_{s}$ and $\boldsymbol{v}$. If a choice is to be made between these two, $v$ is recommended for narrow-band/singlepeaked spectra and $\varepsilon_{s}$ for broad-band/multiple-peaked spectra. Goda's peakedness parameter $Q_{p}$ can be considered to be relatively better as it appears to be independent of the spectral form. Also the estimates of $Q_{p}$ are uncorrelated with the ratio of the highfrequency cut-off to the spectral peak frequency, whereas $\varepsilon_{s}$ and $v$ show an increasing trend with increase in $\mathrm{CF} / f_{p}$.

\section{Acknowledgements}

The author wishes to acknowledge the valuable comments and suggestions offered by the referees, which contributed significantly to this work.

\section{References}

Baba M and Harish C M 1985 Wave height and period distribution off the southwest coast of India; Indian J. Mar. Sci. 14 1-8

Bendat J S and Piersol A G 1971 Random data-analysis and measurement procedures (New York: John Wiley \& Sons)

Cartwright D E and Longuet-Higgins M S 1956 The statistical distribution of the maxima of a random function; Proc. R. Soc, London A237 212-232

Chakrabarti S K and Snider R N 1974 Wave statistics for March 1968 North Atlantic storm; J. Geophys. Res. 79 3449-3458

Dattatri J 1973 Waves off Mangalore harbour, west coast of India; J. Waterways Harbors Div. Am. Soc. Civil Eng. 99 39-58

Dattatri J, Jothi Shankar N and Raman H 1976 Comments on Hoffman and Karst: the theory of the Rayleigh distribution and some of its applications; J. Ship Res. 20 235-238 
Dattatri J, Raman H and Jothi Shankar N 1979 Height and period distributions for waves off Mangalote harbour, west coat of India; J. Geophys. Res. 84 3767-3772

Draper L 1967 The analysis and presentation of wave data-a plea for uniformity; in Proc. 10th Conf. on Coastal Engineering ASCE 1 1-11

Fernandes A A, Gouveia A D, Sathe P V and Nagarajan R 1981 Wave observations in the Mahanadi Basin (Bay of Bengal) during September 1980; Mahasagar, Bull. Natl. Inst. Oceanogr. 14 239-249

Goda T 1970 Numerical experiments on wave statistics with spectral simulation; Rep. Port Harbour Res. Inst. Jpn. 9 3-57

Goda T 1974 Estimation of wave statistics from spectral simulation; in Proc. Intl. Symp. on Ocean Wave Measurement and Analysis, New Orleans

Haring R E, Osborne A R and Spencer L P 1976 Extreme wave parameters based on continental shelf storm wave records; in Proc. 15th Conf. on Coastal Engineering ASCE I 151-170

Hoffman D and Karst OJ 1975 The theory of Rayleigh distribution and some of its applications; J. Ship Res. 19 172-191

John V C 1977 Distributions and relationships of height and period of ocean waves off Mangalore; Indian J. Mar. Sci. 6 49-54

Longuet-Higgins M S 1952 On the statistical distribution of the heights of sea waves; J, Mar. Res. 11 245-266

Longuet-Higgins M S 1975 On the joint distribution of the periods and amplitudes of sea waves; J. Geophys. Res. 80 1688-1694

Narasimhan S and Deo M C 1981 Behaviour of ocean waves at Bombay High and their statistical predictions; First Indian National Conf. on Ocean Engineering, Madras 1981 I 67-74

Prasada Rao C V K 1984 Spectral characteristics of wind-generated ocean gravity waves-a case study; NPOL Dept. Rep. No. RR-1/84 1-16

Prasada Rao C V K 1985 Comparative study of wave parameters derived from spectral and zero-crossing methods; Indian J. Mar. Sci. 14 202-205

Rye H 1976 Discussion of a statistical relationship between individual heights and periods of storm waves; in Proc. 1st Intl. Conf. on Behaviour of Off-shore Structures 2 361-362

Rye $\mathrm{H}$ and Svee R 1976 Parametric representation of a wind-wave field; in Proc. 15th Conf. on Coastal Engineering ASCE I 183-201

Tucker M J 1963 Analysis of records of sea waves; Proc. Inst. Civ. Eng. 26 305-316

Vethamony P, Gopalakrishna V V and Varkey M J 1982 Computation of wave statistics and spectra from records collected with a ship-borne wave recorder off the Godavari Basin (Bay of Bengal) during Sept/Oct 1980; NIO Tech. Rep. No. POD/1/82 1-9

Vethamony P, Gopalakrishna V V and Varkey M J 1984 Wave spectra and statistics off Godavari during Sept/Oct 1980; Mausam 35 199-204 